\title{
AN OPTICALLY LINKED THREE-LOOP ANTENNA SYSTEM FOR DETERMINING THE RADIATION CHARACTERISTICS OF AN ELECTRICALLY SMALL SOURCE
}

\author{
David R. Novotny, Keith D. Masterson, Motohisha Kanda \\ Electromagnetic Fields Division \\ National Institute of Standards and Technology \\ 325 Broadway \\ Boulder, CO 80303
}

\begin{abstract}
This paper presents the experimental results of an antenna system for determining the radiation characteristics of an electrically small source. Three orthogonal loop antennas, each terminated at diametrically opposite points with identical loads, encircle the source and characterize its equivalent electric and magnetic dipole moments. The total radiated power can be determined from this near-field measurement of the device under test. The test system operates from $3 \mathrm{kHz}$ to over $100 \mathrm{MHz}$ with up to $90 \mathrm{~dB}$ of dynamic range.
\end{abstract}

\section{INTRODUCTION}

Electromagnetic radiation (EMR) emitted by equipment may adversely effect other equipment and possibly their users. In particular, the possibility of health hazards to pregnant woman from low-frequency EMR due to video display terminals (VDTs) has received great attention in recent years. This issue has so dominated EMR concerns that the IEEE has formed a standards committee (PAR1140) to study the measurement of VDT radiation from $9 \mathrm{kHz}$ to $50 \mathrm{MHz}$.

Emission characterization of devices using a TEM cell or an open area test site (OATS) measurement are widely used.[1] However, as with most emissions testing techniques, complicated rotations of the device under test (DUT) or of the scanning antenna(s) together with many data points are required to obtain a complete and reliable characterization of the emissions. Such testing can be very time consuming and expensive.

At the National Institute of Standards and Technology (NIST) we have developed a three-loop antenna system (TLAS) that can quickly characterize the dipole radiation patterns from a small device such as a VDT, personal computer, or portable radio transmitter. The TLAS, shown in Fig. 1, consists of three orthogonal loop antennas that are terminated at diametrically opposite points and encircle the DUT and measure its equivalent electric and magnetic dipole moments. The TLAS enables us to quickly characterize the DUT with just six readings from the system and without the necessity of rotating either the device or the loops.

Current testing methods require a far-field measurement done at an OATS or equivalent testing environment.[2] This testing method has many drawbacks, especially at frequencies below 50 $\mathrm{MHz}$, where testing distances are large, signals are low, and ambient noise can be very high. By reducing the measurement distance, this near-field method will allow higher signal levels and greater sensitivity. Similar loop geometries have been proposed to the European Committee for Electrotechnical Standardization (CENELEC) and the International Special Committee on Radio Interference (CISPR)[3] for measuring only magnetic emissions. The TLAS gives a measure of both the electric and magnetic emissions from which the total power radiation can be determined and the far-ficld emission pattern can be estimated.

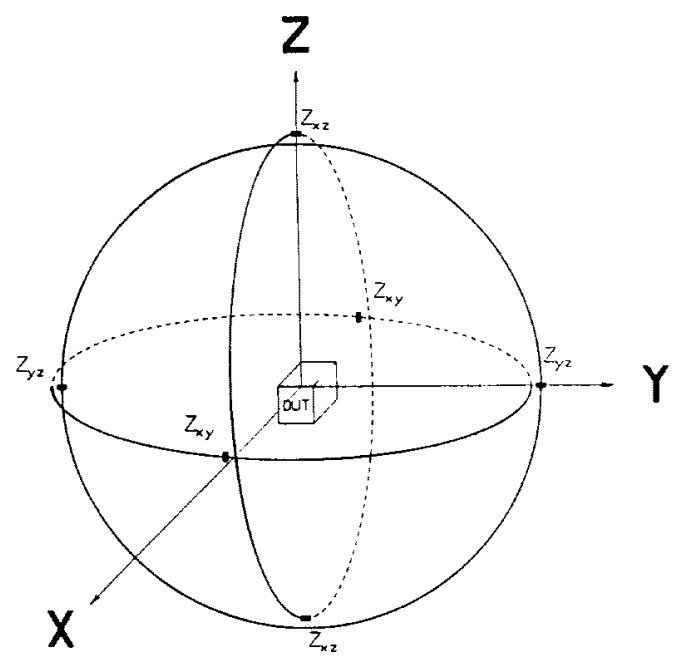

Fig. 1. Geometry of the three-loop antenna system (TLAS) and the placement of a device under test (DUT).

This paper summarizes the theoretical response of the TLAS and describes the construction and test results for a system constructed from three $1 \mathrm{~m}$ diameter loops.

\section{THEORY}

\section{A. Single loop response to an incident plane wave}

Figure 2 shows the geometry and coordinate system of a double gap loop of radius $b$ and conductor radius $a$. The gaps are located at diametrically opposite points $(\phi=0, \pi)$ on the loop and are loaded with identical impedances $Z_{L}$.

Assuming that a linearly polarized wave $E_{o}$ illuminates the loop from the direction $\theta=\pi / 2$ and $\Psi=0$, then following the analysis of Kanda,[4] we obtain the currents at the loads

and

$$
I(0)-2 \pi b E_{o}\left(\frac{f_{0} Y_{0}}{1+2 Y_{0} Z_{L}}+\frac{f_{1} Y_{1}}{1+2 Y_{1} Z_{L}}\right)
$$

$$
I(\pi) \sim 2 \pi b E_{0}\left(\frac{f_{0} Y_{0}}{1+2 Y_{0} Z_{L}}-\frac{f_{1} Y_{1}}{1+2 Y_{1} Z_{L}}\right)
$$

where $f_{0,1}$ are the first two terms of the Fourier series expansion of the plane wave $E_{0}$ as given by Kanda [4], and $Y_{0,1}$ are the admittances seen by the currents induced by the magnetic and electric fields.

\footnotetext{
U.S. Government work not subject to U.S. copyright.
} 


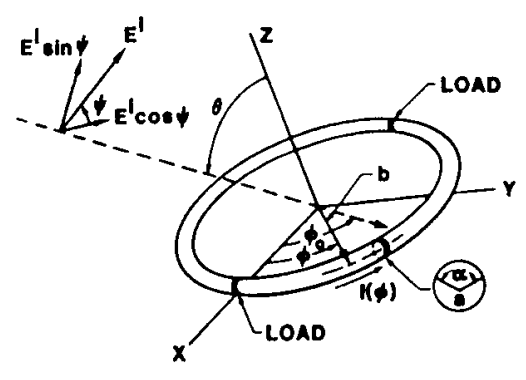

Fig. 2. Configuration of a double gap loop antenna for measuring electric and magnetic fields simultaneously.

by

The quasi-static $(k b<1)$ forms of the admittances are given

$$
Y_{0} \sim \frac{1}{j \eta_{0} k b\left[\ln \left(8 \frac{b}{a}\right)-2\right]}
$$

and

$$
Y_{1} \approx \frac{j 2}{\eta_{0}} \frac{k b}{\left[\ln \left(8 \frac{b}{a}\right)-2\right]}
$$

where $k$ is the wave number and $\eta_{0}$ is the impedance of free space. obtain

By taking the sum and difference of the currents in (1), we

$$
I_{\Sigma} \equiv \frac{1}{2}[I(0)+I(\pi)] \sim 2 \pi b E_{o} \frac{f_{0} Y_{0}}{1+2 Y_{0} Z_{L}}
$$

and

$$
I_{\Delta}=\frac{1}{2}[I(0)-I(\pi)]-2 \pi b E_{o} \frac{f_{1} Y_{1}}{1+2 Y_{1} Z_{L}}
$$

The sum current in (4) depends only on the magnetic component of the plane wave expansion and the admittance seen by the current induced by the magnetic field and thus is proportional to the incident magnetic field. Correspondingly, the difference current in (5) is proportional to the incident electric field. [5]

To optimize the loop response for both the electric and magnetic fields, we equate the sum and difference currents, solve for the load impedance, and conclude:

$$
Z_{L}=\frac{f_{0} Y_{0}-f_{1} Y_{1}}{2 Y_{0} Y_{1}\left(f_{1}-f_{0}\right)}-\eta_{0} \frac{\ln \left(8 \frac{b}{a}\right)-2}{4} .
$$

Equation (6) shows that the optimal loading point of the loop is independent of frequency and dependent only on the ratio of the loop and conductor radii. The theory and experimental data will show that the probe has a wide frequency band in which both the $E$ and $\boldsymbol{H}$ field can be measured simultaneously.

\section{B. Three-loop response to a radiating dipole}

Kanda and Hill[6] have analyzed the response of a system of three orthogonal, concentric loops to a radiating dipole located at the center of the system. Each loop lies in one of the principal rectangular planes with the gaps oriented along a coordinate axis.

Figure 3 shows a loop in the $x y$ plane with a radiating dipole at its center. The load impedances are located on the $x$ axis at $(\phi=0, \pi)$. It has been shown that the currents induced through the loads are given as follows [6]

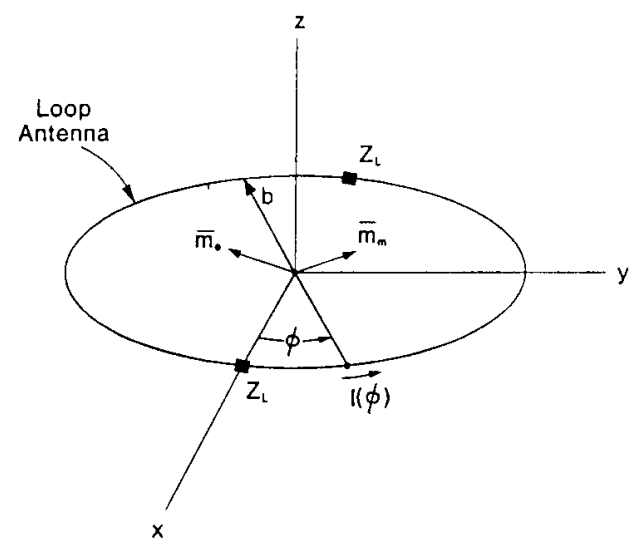

Fig. 3. A double-gap loop antenna excited by electric and magnetic dipoles.

$$
\begin{aligned}
& I_{x y}(0)=2 \pi b\left(\frac{m_{m z} G_{m} Y_{0}}{1+2 Y_{0} Z_{L}}+\frac{m_{e y} G_{e} Y_{1}}{1+2 Y_{1} Z_{L}}\right), \\
& I_{x y}(\pi)=2 \pi b\left(\frac{m_{m z} G_{m} Y_{0}}{1+2 Y_{0} Z_{l}}-\frac{m_{e y} G_{e} Y_{1}}{1+2 Y_{1} Z_{L}}\right),
\end{aligned}
$$

where $m_{m z}$ is the $z$ component of the magnetic dipole moment $m_{m}$, and $m_{e y}$ is the $y$ component of the electric dipole moment $m_{e}$ such that

and

$$
\begin{gathered}
\bar{m}_{m}=m_{m x} \hat{x}+m_{m y} \hat{y}+m_{m z} \hat{z}, \\
\bar{m}_{e}=m_{e x} \hat{x}+m_{e y} \hat{y}+m_{e z} \hat{z} .
\end{gathered}
$$

$\mathrm{G}_{\mathrm{m}}$ and $\mathrm{G}_{\mathrm{e}}$ are given by

and

$$
G_{m}=\frac{\eta_{0}}{4 \pi}\left(\frac{k^{2}}{b}-\frac{j k}{b^{2}}\right) e^{-j k b}
$$

$$
G_{e}=\frac{-\eta_{0}}{4 \pi}\left(\frac{j k}{b}+\frac{1}{b^{2}}+\frac{1}{j k b^{3}}\right) e^{-j k b} \text {. }
$$

By taking the sum and difference of the equations in (7), we obtain the loop response to the magnetic and electric dipole moments as

$$
I_{\Sigma_{x y}}=\frac{1}{2}\left[I_{x y}(0)+I_{x y}(\pi)\right]=2 \pi b \frac{m_{m z} G_{m} Y_{0}}{1+2 Y_{0} Z_{L}}
$$

and

$$
I_{\Delta x}=\frac{1}{2}\left[I_{x y}(0)-I_{x y}(\pi)\right]=2 \pi b \frac{m_{e y} G_{e} Y_{1}}{1+2 Y_{1} Z_{L}}
$$

Equations (10) and (11) show that the sum current is excited by only $m_{m z}$ and the difference currents results from only $m_{e y}$. Analogously, the loop in the $x z$ plane is excited by $m_{m y}$ and $m_{e x}$, and the loop in the $y z$ plane is excited by $m_{m x}$ and $m_{e z}$.

The magnetic and electric dipole moments directly obtained from (10) and (11) are

$$
m_{m z}=\frac{I_{\Sigma_{x y}}\left(1+2 Y_{0} Z_{L}\right)}{2 \pi b G_{m} Y_{0}}
$$

and

$$
m_{e y}=\frac{I_{\Delta_{x y}}\left(1+2 Y_{1} Z_{L}\right)}{2 \pi b G_{e} Y_{1}} .
$$




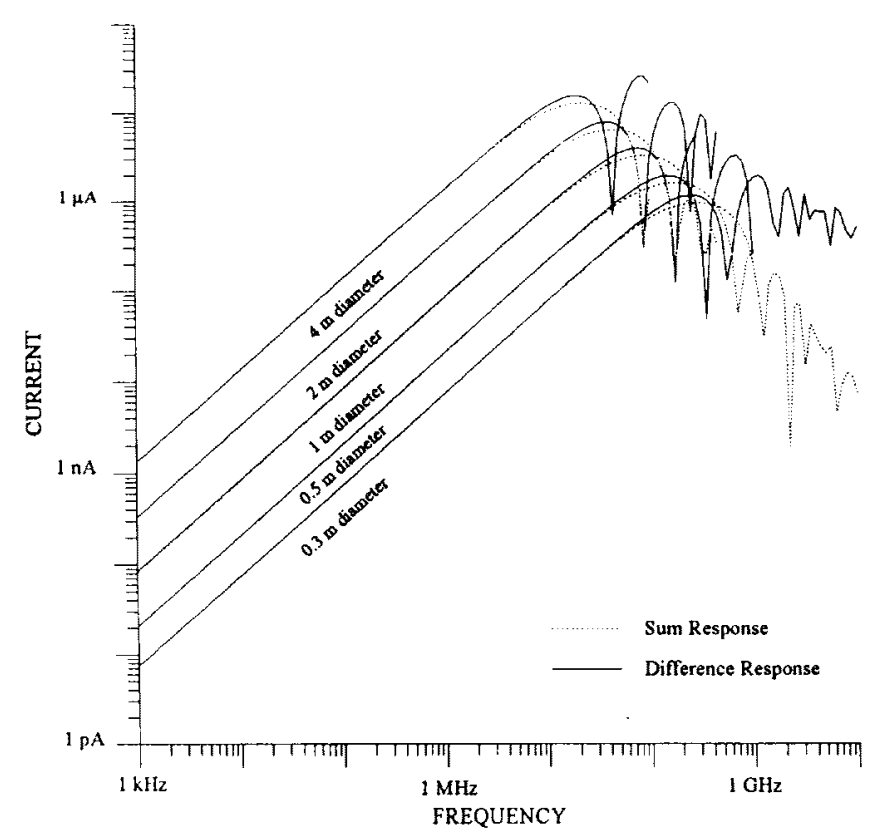

Fig. 4. Sum $\left(I_{\mathrm{E}}\right)$ and difference $\left(I_{\mathrm{L}}\right)$ currents for various antenna diameters. $\left(b / a=26, Z_{L}=315 \Omega, E_{0}=0.3 \mathrm{~V} / \mathrm{m}\right)$

Once the moments are known, it is straightforward [7] to find the net radiated power $P_{T}$

$$
P_{r}=\frac{40 \pi^{2}}{\lambda^{2}}\left[\left|m_{e x}\right|^{2}+\left|m_{e y}\right|^{2}+\left|m_{e z}\right|^{2}+k^{2}\left(\left|m_{m x}\right|^{2}+\left|m_{m y}\right|^{2}+\left|m_{m z}\right|^{2}\right)\right] .
$$

A more complicated expression for the power pattern is given by Koepke, Ma, and Bensema [8] and involves the relative phases and amplitudes of the dipole moments.

\section{ANTENNA AND OPTICAL LINK CONSTRUCTION}

\section{A. Antenna Design}

The curves in Fig. 4 show the theoretical frequency response of various sizes of loops to a linearly polarized $(\Psi=0)$ incident plane wave from the direction $\theta=\pi / 2$. The resonances in the responses are due to higher order modes being excited in the loop and the breakdown of the quasi-static approximation.

The antenna radius, $b$, was chosen as a compromise between a large loop which has high sensitivity and can accommodate a large test object and a small loop which will have a higher cutoff frequency. Since our initial testing was targeted at VDTs, the TLAS had to be large enough to accommodate a VDT and operate up to $100 \mathrm{MHz}$.

The conductor radius, $a$, needs to be kept as small as possible to reduce field perturbations and surface RF current loops. However, to maintain the balanced configuration of the antennas and to prevent ground loops and field perturbations from any lead cables, we put an optical link between the gaps and the remote detector. This necessitated that the loops have a conductor radius large enough to hold the optical transmitter and power source.

Our design of a $1 \mathrm{~m}$ diameter loop was the largest loop which fit the size requirements and operates up to $100 \mathrm{MHz}$. The loops were constructed from $0.019 \mathrm{~m}$ OD copper tubing because of ease of manufacturing, cost, and the internal volume needed for the optical transmitter. The dimensions of the loop and equation (6) leads to an optimal $Z_{L}$ of $315 \Omega$. We chose our load impedance at $290 \Omega$ because the response of the loop is nearly identical for a loading impedance from $270 \Omega$ to $400 \Omega$ and we had a 1:1 balun that operated well to $290 \Omega$.

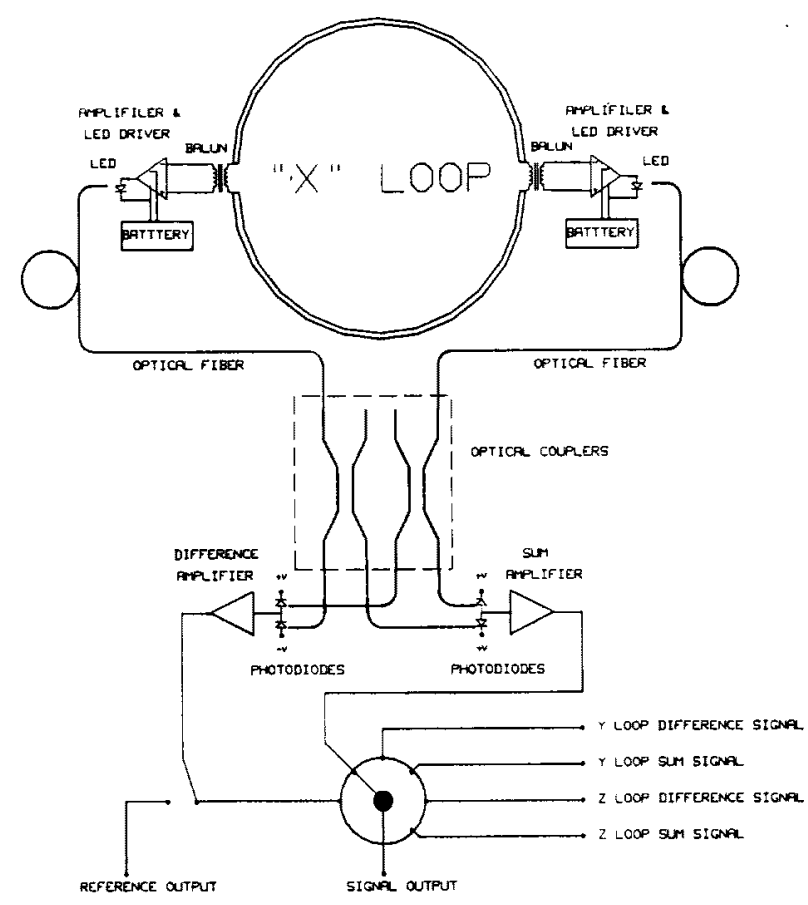

Fig. 5. Block diagram of one loop subsystem of the TLAS together with the output switching diagram.

\section{B. Optical Link Design}

The design of the fiber optic links for each loop was driven by the antenna characteristics. The theoretical curves of Fig. 4 show that the antenna response is linear with frequency. This requires a high dynamic range for both the transmitter and receiver so both ends of the spectrum can have a useful operating range. Since the transmitter and its power source must fit inside the loops, they must be small, have a low component count, and draw relatively little power.

Figure 5 shows the block diagram of one loop of the TLAS. Each transmitter detects one RF gap voltage (current), amplifies it, and modulates an LED. Each optical signal is split by a fiber coupler and detected by a pair of photodiodes in the receiver to create the appropriate sum and difference signals. The components in each loop are carefully matched so the phase and amplitude integrity of the signals are maintained.

The circuitry for each of the optical transmitters and their battery supplies is illustrated in Fig. 6 . The single transistor design was selected because of the low component count and power requirements. The 1:1 balun ensures the antenna remains in a balanced configuration and provides DC isolation between the two transmitter power supplies. The RF current injected into the base of the transistor is amplified and modulates the LED. By adjusting the potentiometer in the base bias circuit, the bias current through the LED can be adjusted. The LED bias current was set so the diode operates in a nearly linear region for a reasonable range of field levels. The base resistor, $R_{b}$, was selected to match the RF impedance of the transistor to the optimal antenna loading impedance. The transistors, LED gain curves, and the balun efficiencies were matched so that the two transmitters in each loop would have modulation responses to within $0.2 \mathrm{~dB}$ of each other and phase difference to within $2^{\circ}$ across the frequency range of interest. While the gain of the transmitter drops off at higher frequencies, the antenna gain increases over frequency resulting in a relatively flat overall response. The battery packs consist of ten AA batteries located inside the loop. They are charged by an external charger 


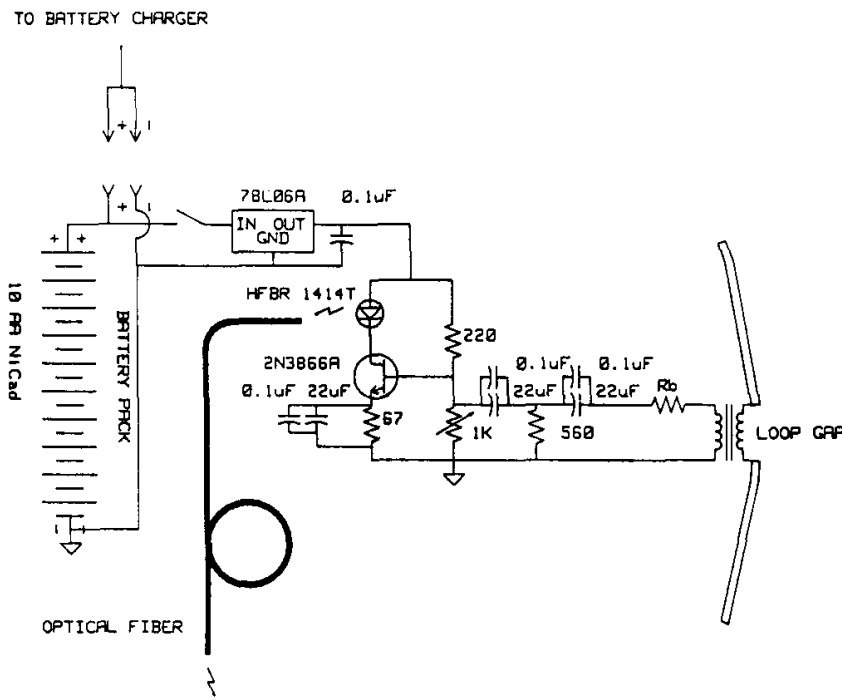

Fig. 6. Schematic of a loop transmitter.

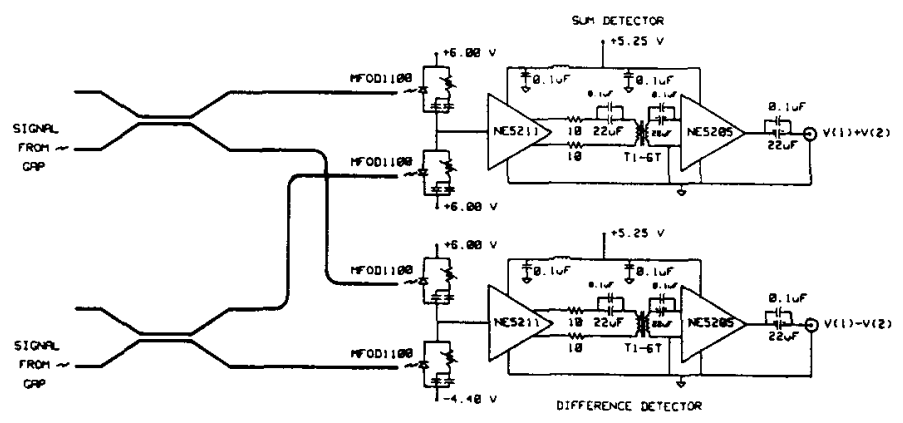

Fig. 7. Schematic of a TLAS reciever circuit.

and, on full charge, can operate for over six hours.

The optical signals are transmitted via a pair of well matched optical fibers to the receiver. The signals from the two gaps in each loop are split by a pair of $3 \mathrm{~dB}$ optical fiber couplers in the receiver. The outputs from each coupler are detected in a way to form the sum and difference signals from each loop. Since the individual gap voltages are combined remotely at the receiver/demodulator, it is imperative that the propagation losses and delays through the links are closely matched. We constructed twelve $15 \mathrm{~m}$ cables and had twelve optical couplers with $1 \mathrm{~m}$ pigtails manufactured, all with a 1 $\mathrm{cm}$ length tolerance. We selected three well matched fiber and coupler pairs for a maximum optical path length difference of 1.5 $\mathrm{cm}$ to keep the phase difference between the signals at $100 \mathrm{MHz}$ within $3^{\circ}$. The response of each transmitter and loss through each fiber link was measured and matched with the gain of a PIN photodiode detector to maintain equal amplitude responses for each pair of loop signals throughout the system.

The receiver is made of two independent circuits as shown in Fig. 7. The sum and difference of the gap signals are obtained by the configuration of the photodiodes with respect to the transimpedance amplifiers. In the sum circuit, the incident light in diodes D1 and D2 both sink RF current from the transimpedance amplifier which yields a net sum of the currents. In the difference circuit, diode D3 sinks RF current, while diode D4 sources RF current from the transimpedance amplifier so the output is proportional to the difference of the signals. In both circuits, the balanced output of the transimpedance amplifier is converted to an unbalanced signal which is further amplified by a MMIC amplifier that provides additional gain and isolation of the detector circuit from the other output signals.
The TLAS consists of three sets of transmitters, optical links, and receivers. The output is selectable from any of the six sum and difference outputs, or the $x y$ loop sum current may be used as a reference and the other signals may be compared to it for relative phase and amplitude information.

With the use of a high quality $0 / 180$ hybrid, we injected signals into the transmitters and measured the performance of the system across the $1 \mathrm{kHz}$ to $150 \mathrm{MHz}$ bandwidth. When equal out of phase signals were added or equal in phase signals subtracted, a minimum of $25 \mathrm{~dB}$ of nulling was achieved across the $3 \mathrm{kHz}$ to 100 $\mathrm{MHz}$ bandwidth. The sum of in phase or the difference of out of phase signals provided a minimum of $5.8 \mathrm{~dB}$ increase in the output over a single input when the two equal amplitude signals were combined.

By injecting a known signal into the transmitters and measuring each output, we compiled a set of calibration curves of the ratio of gap current to receiver output voltage for each sum and difference output.

\section{EXPERIMENTAL EVALUATION}

\section{A. Single-Loop Results}

We tested the plane wave response of a single loop in a TEM cell. We used a $1 \mathrm{~m}$ diameter loop with a gap loading of $290 \Omega$. The cell had a septum to wall separation of $1.5 \mathrm{~m}$ and with the loop centered in the cell, we could neglect the fringing fields near the cell walls. The loop was illuminated with a $0.3 \mathrm{~V} / \mathrm{m}$ field, and the calibration data were applied to the sum and difference outputs. The experimentally determined loop responses are plotted with the theoretically calculated values in Fig. 8. The difference between experiment and theory is less than $1.5 \mathrm{~dB}$ from $20 \mathrm{kHz}$ to $20 \mathrm{MHz}$ and is less than $4 \mathrm{~dB}$ at $3 \mathrm{kHz}$. The $20 \mathrm{MHz}$ frequency limit in the test data is due to the TEM cell limitations.

The linearity of the antenna at $1 \mathrm{MHz}$ and the range of operation were measured and plotted in Fig. 9. The TLAS shows $90 \mathrm{~dB}$ of dynamic range at a $10 \mathrm{~Hz}$ measurement bandwidth. To confirm the sum and difference pattern characteristics and to show that, in the TLAS, each vector component of the dipole moments will only induce one sum or difference current, the single loop was rotated about an axis parallel to the $E$ field in the TEM cell. The

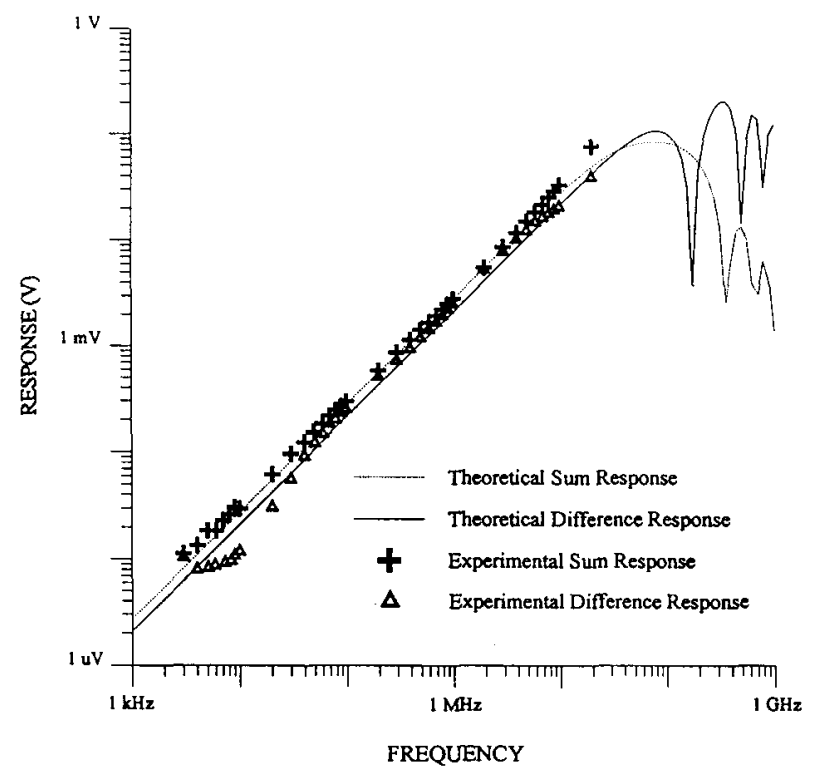

Fig. 8. Plane wave response of a double gap loop antenna. $\left(E_{0}=0.3 \mathrm{~V} / \mathrm{m}, Z_{L}=290 \Omega\right)$ 


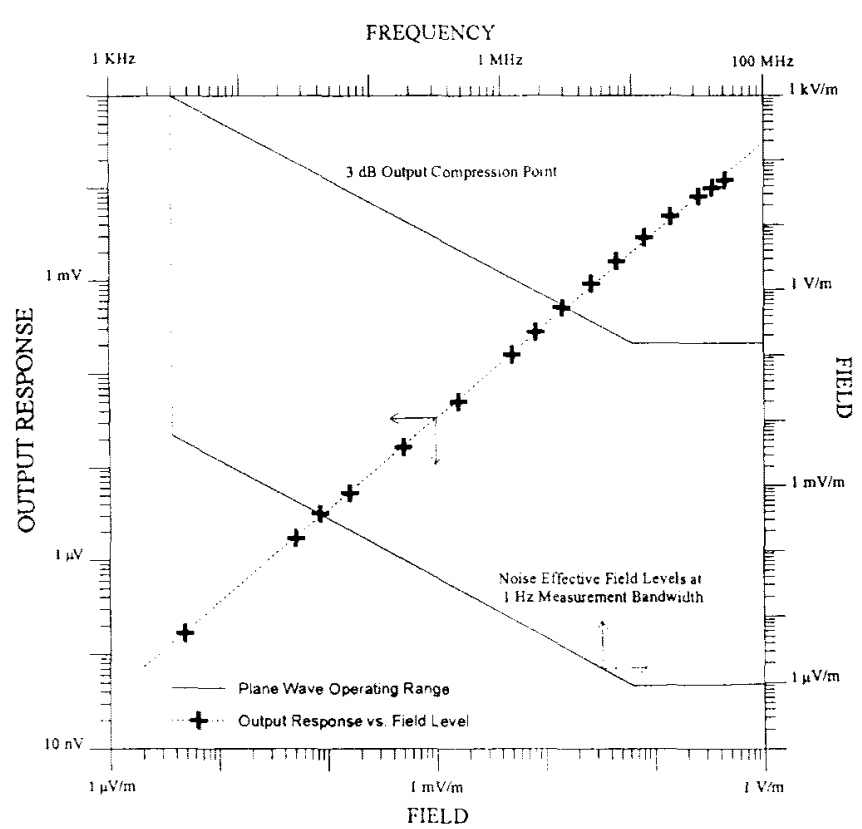

Fig. 9. Linearity (at $1 \mathrm{MHz}$ ) and plane wave operating range of a double gap loop antenna.

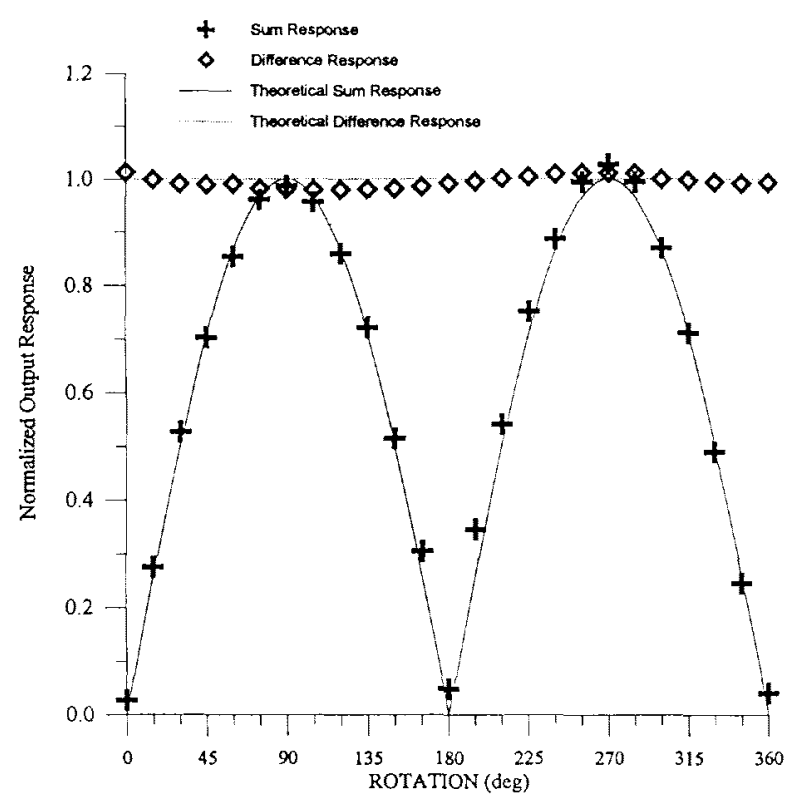

Fig. 10. Response pattern of a double gap loop antenna determined by rotating the antenna in a TEM at $5 \mathrm{MHz}$.

results of the rotation (Fig. 10) confirm equations (10) and (11) by showing that the sum current is proportional to the vector projection of the magnetic field and the vector normal to the plane of the loop ( $z$ axis of Fig. 2). A similar rotation about the axis of the gaps show the difference current is proportional to the vector projection of the electric field and $y$ axis in Fig. 2.

\section{B. Three-Loop Antenna System (TLAS) Results}

The TLAS was setup as shown in Fig. 11 to measure the total power radiated from a test object. The tests were conducted at the NIST OATS, a $30 \mathrm{~m} \times 60 \mathrm{~m}$ flat ground screen. The DUT was a $10 \mathrm{~cm}$ diameter, standard spherical dipole radiator (SDR)[9] developed and calibrated at NIST. The SDR consists of two metal

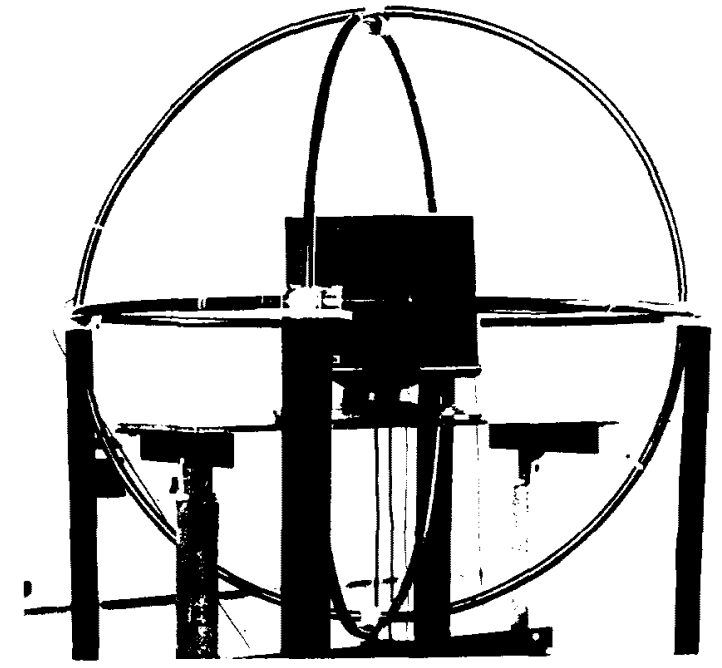

Fig. 11. The TLAS for determining the radiation characteristics of an electrically small source.

hemispheres separated by a thin gap across which a RF voltage is applied and measured. The SDR closely models an ideal electric dipole radiator at frequencies below $100 \mathrm{MHz}$, and its electric dipole moment is oriented perpendicular to the gap.

We placed the SDR at the center of the TLAS and measured the sum and difference outputs from each loop. The results of the radiated power measurements made using the TLAS are shown in Fig. 12 and compared to the calculated power radiated by the sphere operating at the same gap voltage during previous far-field calibrations at OATS. The power radiated by the electric dipole moment of the SDR, as measured by the TLAS, is over $20 \mathrm{~dB}$ greater than the power radiated by the magnetic dipole. There is an approximate $8 \mathrm{~dB}$ discrepancy between the OATS far-field data and the TLAS data. Previous testing in a TEM cell showed that the SDR was susceptible to loading by the cell. To determine whether the loops were having a similar effect on the SDR, we recalibrated the SDR's far-field pattern with and without the loop system encircling it. Between 30 to $90 \mathrm{MHz}$, we calculated a 6 to $7 \mathrm{~dB}$ reduction in the measured radiated power when the SDR was inside of the loop system. When we corrected the initial measurements by this factor the results agreed with the far-field calculations to within

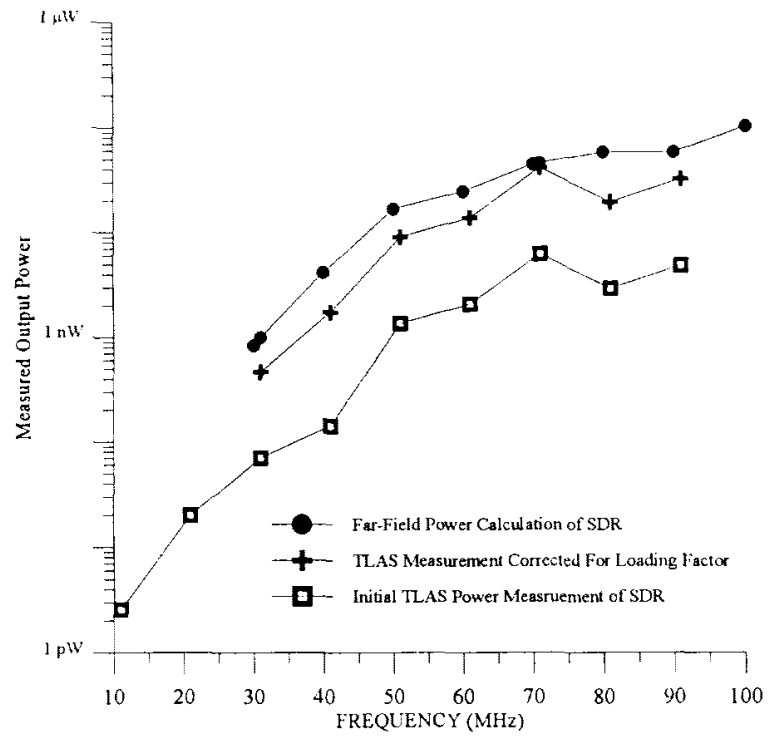

Fig. 12. Comparison of the Spherical Dipole Radiator's (SDR) far field power radiation characteristics and the TLAS measurements. 


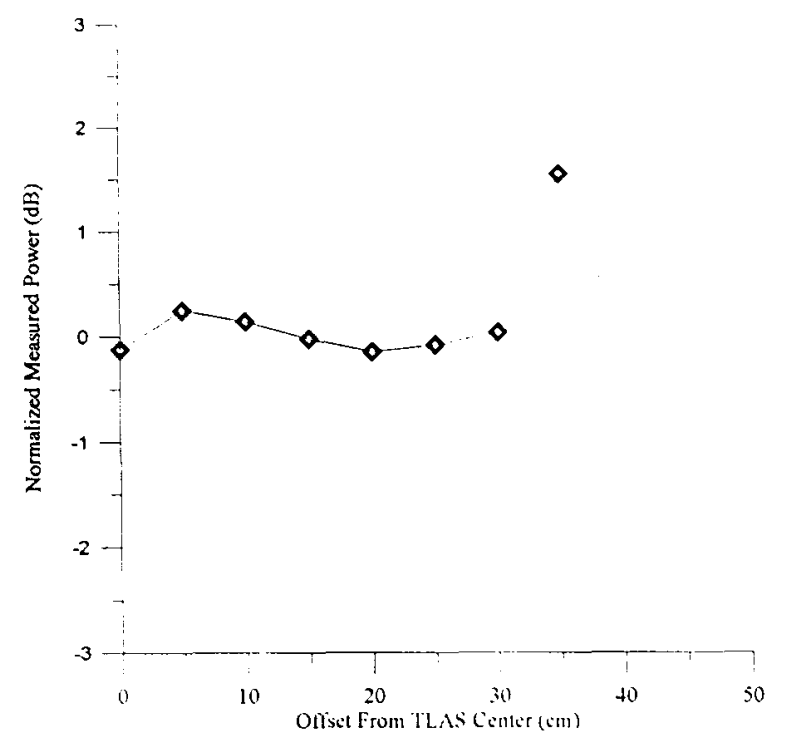

Fig. 13. Calculated power measurements of the SDR vs. position within the TLAS.

the measurement uncertainty. The details and consequences of the coupling between a DUT and an antenna in the near-field (such as a TEM cell or TLAS) will be more thoroughly explored in the future.

The SDR output was measured at various rotations and positions within the interior of the loops at 30 and $70 \mathrm{MHz}$. The total measured output power was independent of rotation and the measured vector components of the dipole moments followed the rotation of the SDR. The results of readings taken throughout the interior of the loop system are shown in Fig. 13. It is determined that the largest dimension of a DUT would be limited to $60 \mathrm{~cm}$ (or $30 \mathrm{~cm}$ from the center of the TLAS) in order for the measured output pattern to agree with theory. When the SDR was located 30 to $38 \mathrm{~cm}$ from the center of the TLAS, the degradation in the calculated radiated power was still less than $3 \mathrm{~dB}$. However when the dipole was further than $30 \mathrm{~cm}$ from the center of the loops, the measured dipole pattern no longer corresponded to the ideal dipole pattern of the SDR in free space. This shows that exact placement of the DUT is not critical to loop operation. If larger devices require testing or greater sensitivity is desired at lower frequencies, the loops may be increased in size with the loss in bandwidth at the higher frequencies of operation.

\section{MEASUREMENT UNCERTAINTIES}

The plane wave tests, frequency response, linearity and rotation, have a measurement uncertainty of $\pm 1.5 \mathrm{~dB}$. This uncertainty results primarily from errors in alignment of the loop within the TEM cell.

The uncertainties for the OATS far-field data on the SDR are $\pm 2 \mathrm{~dB}$. The measured results for the frequency response of the TLAS are accurate to within $\pm 2.5 \mathrm{~dB}$. These errors arise from the uncertainty in the calibration of the far-field data for the sphere, the uncertainty in the calculation of the loading factor of the spheres, and the calibration data for the TLAS. The measurement uncertainties for the scan of the interior of the loops is $\pm 1.5 \mathrm{~dB}$.

\section{ACKNOIVLEDGENIENIS}

We thank the following people for their contributions to the project: Kenneth Cavcey for his help in circuit design; Robert Johnk for his help in the practical EM analysis of the loops and RF analysis of the optical receiver; Dean Melquist and Victor Lombardo for their help in construction of the loops; Galen Keopke, R. David Orr, and Arthur Ondrejka for their help in measurement analysis, data gathering and testing procedures; and finally, Carl Stubenrauch for his insights into the loop loading phenomena.

\section{REFERENCES}

[1] I. Sreenivasiah, D. C. Chang, and M.T. Ma, "A Method of Determining the Emission and Susceptibility Levels of Electrically Small Objects Using a TEM Cell," National Bureau of Standards (U.S.A.) Technical Note 1040, Apr. 1981.

[2] Federal Communications Commission, FCC Procedure for Measuring RF Emissions from Computing Devices FCC/OET MP-4 (1987). July 1987, pp. 3-5.

[3] J.R. Bergervoet and H.V. Veen, "A Large Loop Antenna For Magnetic Field Measurements, " presented at The 8th Int. Zurich Symposium and Technical Exhibition on Electromagnetic Compatibility, Zurich, Mar. 7-9, 1989, pp. 29-34.

[4] M. Kanda, "An Electromagnetic Near-Field Sensor for Simultaneous Electric and Magnetic-Field Measurements," IEEE Transactions on Electromagnetic Compatibility, Vol. 26, No. 3, Aug 1984, pp. 102-110.

[5] L.D. Driver, M. Kanda, "An Optically Linked Electric and Magnetic Field Sensor for Poynting Vector Measurements in the Near Fields of Radiating Sources, "IEEE Transactions on Electromagnetic Compatibility, Vol.30, No. 4, Nov 1988.

[6] M. Kanda and D.A. Hill, "A Three-Loop Method for Determining the Radiation Characteristics of an Electrically Small Source," IEEE Transactions on Electromagnetic Compatibility, Vol. 34, No. 1, Feb 1992, pp. 1-3.

[7] R.F. Harrington, Time-Harmonic Electromagnetic Fields. New York: McGraw-Hill, 1961.

[8] G.H. Keopke, M.T. Ma and W.D. Bensema, "Theory and Measurements of Radiated Emissions Using a TEM Cell," National Institute of Standards and Technology Technical Note 1326, Jan. 1989.

[9] G. Keopke, L.D. Driver, K. Cavcey, K. Masterson, R. Johnk, M. Kanda, "Standard Spherical Dipole Source," National Institute of Standards and Technology Technical Note 1351, Dec 1991. 